ON QUASIPERIODIC OSCILLATIONS OF

\title{
A NONLINEAR DYNAMIC SYSTEM OF LIAPUNOV TYPE WITH TIME LAG
}

\author{
LE XUAN CAN \\ Department of Mechanics - VNU Hanoi
}

\begin{abstract}
The paper is concerned with the investigation of the quasiperiodic oscillations of a nonlinear dynamic system of Liapunov type with time lag. The following results are obtained:

- The necessary and sufficient conditions for the existence of the quasiperiodic solution describing the oscillating processes.

- The approximate quasiperiodic solution in the power series.

- The quasiperiodic oscillations of a nonlinear dynamic system of Duffing type with the quasiperiodic perturbations.
\end{abstract}

1. Let us consider a nonlinear dynamic systen described by the differential equaion of the form

$$
\frac{d^{2} x}{d t^{2}}+\omega^{2} x+X(x)=\varepsilon F\left(t, x, x_{\Delta}, \frac{d x}{d t}, \frac{d x_{\Delta}}{d t}\right)
$$

where $\varepsilon$ is a small parameter, $X(x)$ is a power series in $x$ of the form

$$
X(x)=\gamma_{2} x^{2}+\gamma_{3} x^{3}+\ldots
$$

$F$ is a continuous in $t$ and quasiperiodic function with frequency basis $\nu_{1}, \nu_{2}, \ldots, \nu_{n}$ Ind analytical function in $x, x_{\Delta}, \frac{d x}{d t}, \frac{d x_{\Delta}}{d t}$ in the domair $D$. Here $x_{\Delta}=x(t-\Delta)$, $\frac{d x_{\Delta}}{d t}=\frac{d}{d t} x(t-\Delta), \Delta$ is a positive number.

Together with given differential equation we consider the following differential :quation called degenerate equation, which can be obtained from (1.1) by putting $:=0$ :

$$
\frac{d^{2} x}{d t^{2}}+\omega^{2} x+X(x)=0
$$


It is easy to see that equation (1.3) is differential equation of Liapunov type because we can find its initial integral in the form

$$
\left(\frac{d x}{d t}\right)^{2}+\omega^{2} x^{2}+2 \int X(x) d x=\text { const. }
$$

It is known that the Liapunov type equation (1.3) has a continuous in $t$ and periodic solution depending on two parameters with period $T$ in the form [1]

$$
T=\frac{2 \pi}{\omega}\left(1+\alpha_{2} c^{2}+\alpha_{3} c^{3}+\ldots\right)
$$

where the first coefficient $\alpha_{i}$ in expression (1.5) is different from zero and has the even index (denoted by $\alpha_{i}^{*}$ ), $c$ is value of $x$ in initial moment $t=0$.

We prove first the following theorem:

Theorem 1.1. If the development of function $X(x)$ in power series is such that the first coefficient $\gamma_{i}$ which is different from zero and denoted by $\gamma_{i}^{*}$ has the odd index $2 m+1$, then the coefficient $\alpha_{i}^{*}$ has even index $2 m$. If $\gamma_{i}^{*}$ has even index $2 m$ and following odd index is equal to $2 m+2 k+1(k=0,1,2, \ldots)$ for $\gamma_{j}=0$, then $\alpha_{i}^{*}$ has index $\min (2 m+2 k, 4 m-2)$.

Proof. Making in the equation (1.3) the variable change $t=(1+\alpha) \frac{\tau}{\omega}$ we obtain the following equation:

$$
\frac{d^{2} x}{d t^{2}}+x+\alpha(2+\alpha) x+\left(\frac{1+\alpha}{\omega}\right)^{2} X(x)=0 .
$$

Together with (1.6) we consider an auxiliary integro-differential equation of the form

$$
\begin{aligned}
& \frac{d^{2} x}{d \tau^{2}}+x+\alpha(2+\alpha) x+\left(\frac{1+\alpha}{\omega}\right)^{2} X(x)=W \cos \tau \\
& W=\frac{1}{\pi} \int_{0}^{2 \pi}\left[\alpha(2+\alpha) x+\left(\frac{1+\alpha}{\omega}\right)^{2} X(x)\right] \cos \tau d \tau .
\end{aligned}
$$

The continuous in $\tau$ and $2 \pi$-periodic solution of the system (1.7) will be found by the iteration method with the initial conditions $x(0)=c, \frac{d x(0)}{d \tau}=0$.

As the 0-th order approximation for this solution we take:

$$
\left\{\begin{array}{l}
x_{0}(\tau)=c \cdot \cos \tau \\
W_{0}=0
\end{array}\right.
$$


As the $n$-th order approximation for the solution we shall take $\imath ! 2 \pi$-periodic solution of the following nonhomogeneous linear system of equation:

$$
\begin{gathered}
\frac{d^{2} x_{n}}{d \tau^{2}}+x_{n}+\alpha(2+\alpha) x_{n-1}+\left(\frac{1+\alpha}{\omega}\right)^{2} X\left(x_{n-1}\right)=W_{n} \\
W_{n}=\frac{1}{\pi} \int_{0}^{2 \pi}\left[\alpha(2+\alpha) x_{n-1}+\left(\frac{1+\alpha}{\omega}\right)^{2} X\left(x_{n-1}\right)\right] \cos \tau d \tau,(n=; \ldots, \ldots) .
\end{gathered}
$$

It has the form

$$
x_{n}(\tau)=M \cos \tau+L\left[\tau, \alpha(2+\alpha) x_{n-1}+\left(\frac{1+\alpha}{\omega}\right)^{2} X\left(x_{n-1}\right)-W, \cdots,\right],
$$

where $M$ will be determined by initial conditions, operator $L[\tau, y(\tau)]$ is linear and homogeneous in $y(\tau)$ and admits estimate $|L[\tau, y(\tau)]|<A B$ with $\max |y(\tau)| \leq B$ and with constant $A$ which does not depend on $y(\tau)$.

Carrying out the estimate of the quantities $\left|x_{n}(\tau)-x_{0}(\tau)\right|,\left|W_{n}\right|, \mid x_{n}(\tau)-$ $x_{n-1}(\tau)|,| W_{n}-W_{n-1} \mid$ we can assert that the sequences $\left\{x_{n}(\tau)\right\},\left\{W_{n}\right\}$ are uniformly convergent to the limit functions $\bar{x}(\tau, c, \alpha), \bar{W}(c, \alpha)$ with enough small $c$.

These functions satisfy equation (1.7) and $\bar{x}(\tau, c, \alpha)$ is continuous in $\tau$ and $2 \pi$ periodic function and analytical in $c$ and $\alpha$ function.

We can express the function $\bar{W}(c, \alpha)$ in the form

$$
\bar{W}(c, \alpha)=\alpha(2+\alpha)\left(c-c^{2 \beta+1} \delta_{1}-\ldots\right)+c^{2 \beta+1}\left(\delta_{2}+c(\ldots)\right)
$$

where $\delta_{1}, \delta_{2}$ are constants, $\delta_{2} \neq 0,2 \beta=2 m$ if $\gamma_{i}^{*}$ has the odd index $2 m+1$, $2 \beta=\min (2 m+2 k, 4 m-2)$ if $\gamma_{i}^{*}$ has the even index $2 m$ and following odd index with $\gamma_{j} \neq 0$ is equal to $2 m+2 k+1$. Hence

$$
\bar{W}(c, \alpha)=0
$$

is the necessary and sufficient conditions for the existence of the continuous in $\tau$ and $2 \pi$-periodic solution of the equation (1.6).

Developing left-hand side of equality (1.12) in the power series in $\alpha$ we find

$$
\alpha=\alpha_{0} e^{2 \beta}+\ldots
$$

In the particular case: $2 m+2 k=4 m-2$ and the coefficients for correspondent degree in the expression (1.11) are the same on modulus and are inverse on the 
sign, the development of $\alpha(c)$ in the power series will be begun by the term of highest order greater than $\min (2 m+2 k, 4 m-2)$.

However, this case can be eliminated with the aid of variation of coefficient $\gamma_{2 m+2 k+1}$.

2. In this section we consider the differential equation of the form (1.1). Suppose that there exists the combinatory resonance in the dynamic system, i.e. there is the relationship:

$$
\omega=k_{1} \nu_{1}+k_{2} \nu_{2}+\cdots+k_{n} \nu_{n}=(\mathbf{k}, \boldsymbol{\nu})
$$

where $\mathbf{k}=\left(k_{1}, k_{2}, \ldots, k_{n}\right), \boldsymbol{\nu}=\left(\nu_{1}, \nu_{2}, \ldots, \nu_{n}\right),(\mathbf{k}, \boldsymbol{\nu})$ is scalar product of two vectors $\mathbf{k}$ and $\boldsymbol{\nu}$.

The purpose of this section is to give the necessary and sufficient conditions for the existence of the continuous in $t$ and quasiperiodic solution with the frequency basis $\nu_{1}, \nu_{2}, \ldots, \nu_{n}$ of the differential equation (1.1). We shall present again the method for construction of the approximate solution of the equation in the power series.

For this aim we introduce the partial differential equation called associated equation in the form [2].

$$
\sum_{i, j=1}^{n} \frac{\partial^{2} u}{\partial \theta_{i}} \nu_{i} \nu_{j}+(\mathbf{k}, \boldsymbol{\nu})^{2} \cdot u+Y(u)=\varepsilon \Phi\left(\theta_{1}, \ldots, \theta_{n}, u, u_{\Delta}, \sum_{i=1}^{n} \frac{\partial u}{\partial \theta_{i}} \nu_{i}, \sum_{i=1}^{n} \frac{\partial u_{\Delta}}{\partial \theta_{i}} \nu_{i}\right)
$$

where $Y(u)=X(x=u), u=u\left(\theta_{1}, \ldots, \theta_{n}, \varepsilon\right)$

$$
\begin{gathered}
\Phi=F\left(\nu_{i} t=\theta_{i}, x=u, x_{\Delta}=u_{\Delta}, \frac{d x}{d t}=\sum_{i=1}^{n} \frac{\partial u}{\partial \theta_{i}} \nu_{i}, \frac{d x_{\Delta}}{d t}=\sum_{i=1}^{n} \frac{\partial u_{\Delta}}{\partial \theta_{i}} \nu_{i}\right) \\
u_{\Delta}=u\left(\theta_{1}-\nu_{1} \Delta, \ldots, \theta_{n}-\nu_{n} \Delta, \varepsilon\right) .
\end{gathered}
$$

We can easy assert

Theorem 2.1. If the function $u\left(\theta_{1}, \ldots, \theta_{n}, \varepsilon\right)$ is the continuous in $\theta_{1}, \theta_{2}, \ldots, \theta_{n}$ and $2 \pi$-periodic solution of the equation (2.2), then the function $x(t, \varepsilon)=$ $u\left(\nu_{1} t, \ldots, \nu_{n} t, \varepsilon\right)$ is the continuous in $t$ and quasiperiodic solution with frequency basis $\nu_{1}, \nu_{2}, \ldots, \nu_{n}$ of differential ordinary equation (1.1).

The proof is straightforward. 
To the equation (2.2) we associate now an auxiliary system of integro-differential equations of the form

$$
\begin{aligned}
& \sum_{i, j=1}^{n} \frac{\partial^{2} \nu}{\partial \theta_{i} \partial \theta_{j}} \nu_{i} \nu_{j}+(\mathbf{k}, \boldsymbol{\nu})^{2} \boldsymbol{\nu}+Y(\boldsymbol{\nu})= \\
& =\varepsilon \Phi\left(\theta_{1}, \ldots, \theta_{n}, \nu, \nu_{\Delta}, \sum_{i=1}^{n} \frac{\partial \nu}{\partial \theta_{i}} \nu_{i}, \sum_{i=1}^{n} \frac{\partial \nu_{\Delta}}{\partial \theta_{i}} \nu_{i}\right)+W e^{i(\mathbf{k}, \theta)}+\bar{W} e^{-i(\mathbf{k}, \theta)} \\
& W=\frac{1}{(2 \pi)^{n}} \int_{0}^{2 \pi} \ldots \int_{0}^{2 \pi}[Y(\boldsymbol{\nu})-\varepsilon \Phi] e^{-i(\mathbf{k}, \theta)} d \theta_{1} \ldots d \theta_{n}
\end{aligned}
$$

where $\bar{W}$ is complex conjugate quantity for $W,(\mathbf{k}, \theta)$ is scalar product of two vectors $\mathbf{k}=\left(k_{1}, \ldots, k_{n}\right),=\left(\theta_{1}, \ldots, \theta_{n}\right)$.

Theorem 2.2. The system of integro-differential equations (2.3) has always a family of continuous in $\theta_{1}, \theta_{2}, \ldots, \theta_{n}$ and $2 \pi$-periodic solutions depending on two arbitrary constants.

Proof. The theorem will be proved by the iteration method. As the 0-th order approximation of the solution of the system (2.3) we take

$$
\begin{aligned}
\nu_{0} & =a e^{i(k, \theta)}+\bar{a} e^{-i(\mathbf{k}, \theta)}, \\
W_{0} & =0
\end{aligned}
$$

where $a$ is arbitrary constant, $\bar{a}$ is complex conjugate quantity for $a$.

As the $n$-th approximation of the solution of the system (2.3) we take the solution of nonhomogeneous linear system of partial differential equations of the form

$$
\begin{gathered}
\sum \frac{\partial^{2} \nu_{n}}{\partial \theta_{i} \partial \theta_{j}} \nu_{i} \nu_{j}+(\mathbf{k}, \nu)^{2} \nu_{n}+Y\left(\nu_{n-1}\right)=\varepsilon F_{n-1}+W_{n} e^{i(\mathbf{k}, \theta)}+\bar{W}_{n} e^{-i(\mathbf{k}, \theta)} \\
W_{n}=\frac{1}{(2 \pi)^{n}} \int_{0}^{2 \pi} \ldots \int_{0}^{2 \pi}\left[Y\left(\nu_{n-1}\right)-2 F_{n-1}\right] e^{-i(\mathbf{k}, \theta)} d \theta_{1} \ldots d \theta_{n}
\end{gathered}
$$

where

$$
F_{n-1}=F\left[\theta_{1}, \ldots, \theta_{n}, \nu_{n-1}, \nu_{\Delta, n-1}, \sum_{i=1}^{n} \frac{\partial \nu_{n-1}}{\partial \theta_{i}} \nu_{i}, \sum \frac{\partial \nu_{\Delta, n-1}}{\partial \theta_{i}} \nu_{i}\right]
$$


Setting the estimate on the quantities $\left|\nu_{n}-\nu_{n-1}\right|,\left|W_{n}-W_{n-1}\right|,\left|\nu_{n}-\nu_{0}\right|$, $\left|W_{n}\right|$ we can assert that the sequences $\left\{\nu_{n}\right\},\left\{W_{n}\right\}$ are uniformly convergent to the limit functions $\nu_{*}, W_{*}$ with enough small $\varepsilon$ and presented boundary conditions.

$\nu_{*}$ and $W_{*}$ satisfy the system of equations (2.3), the function depending on two arbitrary constants $a_{r}$ and $a_{i m}\left(a_{r}=\operatorname{Re} a, a_{i m}=\operatorname{Im} a\right)$.

From this result we can deduce the following theorem:

Theorem 2.3. The necessary and sufficient conditions for the existence of the continuous in $t$ and quasiperiodic solution with the frequency basis $\nu_{1}, \ldots, \nu_{n}$ of equation (1.1) are that the constant $a_{r}$ and $a_{i m}$ satisfy the following equalities:

$$
\text { at } \quad \begin{aligned}
& \operatorname{Re} W=0, \\
& \operatorname{Im} W=0 .
\end{aligned}
$$

Going into details we can show that if the function $X(x)$ is a power series in $x$ in the form

$$
X(x)=\gamma_{2 m+1} x^{2 m+1}+\gamma_{2 m+2} x^{2 m+2}+\ldots
$$

then the quantities $a_{r}$ and $a_{i m}$ can be expressed in the form

$$
\begin{aligned}
a_{r} & =a_{11} \varepsilon^{\frac{1}{2 m+1}}+a_{12} \varepsilon^{\frac{2}{2 m+2}}+\ldots, \\
a_{i m} & =a_{21} \varepsilon^{\frac{1}{2 m+1}}+a_{22} \varepsilon^{\frac{2}{2 m+2}}+\ldots
\end{aligned}
$$

Giving $\varepsilon \rightarrow 0$ the continuous in $t$ and quasiperiodic solution obtained with frequency basis $\nu_{1}, \nu_{2}, \ldots, \nu_{n}$ of differential equation (1.1) deduces to trivial solution $x=0$ of degenerate equation (1.3).

3. As an application of the presented method we consider a differential equation describing the oscillations in a nonlinear dynamic system of Duffing type under the quasiperiodic perturbations:

$$
\frac{d^{2} x}{d t^{2}}+\omega^{2} x+\gamma x^{3}=\varepsilon\left[\left(1-\alpha x_{\Delta}^{2}\right) x_{\Delta}+A_{1} \cos \nu_{1} t+A_{2} \cos \nu_{2} t\right]
$$

where $\gamma, \alpha, \Delta, A_{1}, A_{2}$ are positive constants, $\varepsilon$ is a small parameter, $\nu_{1}$ and $\nu_{2}$ are independent, i.e. $k_{1} \nu_{1}+k_{2} \nu_{2}=0$ and $k_{1}, k_{2}$ - integers imply $k_{1}=0, k_{2}=0$. We assume that $\omega=\nu_{1}$, i.e. there exists the principal resonance on the frequency $\nu_{1}$. 
Associated equations for (3.1) can be written in the form

$$
\begin{aligned}
\left(\frac{\partial}{\partial \theta_{1}} \nu_{1}\right)^{2} u+\nu_{1}^{2} u+\gamma u^{3}= & \varepsilon\left[\left(1-\alpha u_{\Delta}^{2}\right)\left(\frac{\partial \nu_{\Delta}}{\partial \theta_{1}} \nu_{1}+\frac{\partial \nu_{\Delta}}{\partial \theta_{2}} \nu_{2}\right)\right. \\
& \left.+A_{1} \cos \theta_{1}+A_{2} \cos \theta_{2}\right]
\end{aligned}
$$

and corresponding auxiliary system can be expressed in the form

$$
\begin{aligned}
& \left(\frac{\partial}{\partial \theta_{1}} \nu_{1}+\frac{\partial}{\partial \theta_{2}} \nu_{2}\right)^{2} \nu+\nu_{1}^{2} \nu+\gamma \nu^{3}= \\
& =\varepsilon\left[\left(1-\alpha \nu_{\Delta}^{2}\right)\left(\frac{\partial \nu_{\Delta}}{\partial \theta_{1}} \nu_{1}+\frac{\partial \nu_{\Delta}}{\partial \theta_{2}} \nu_{2}\right)+A_{1} \cos \theta_{1}+A_{2} \cos \theta_{2}\right]+W e^{i \theta_{1}}+\bar{W} e^{-i \theta_{1}} \\
& W=\frac{1}{(2 \pi)^{2}} \int_{0}^{2 \pi} \int_{0}^{2 \pi}\left[\gamma \nu^{3}-\varepsilon\left(1-\alpha \nu_{\Delta}^{2}\right)\left(\frac{\partial \nu_{\Delta}}{\partial \theta_{1}} \nu_{1}+\frac{\partial \nu_{\Delta}}{\partial \theta_{2}} \nu_{2}\right)\right. \\
& \left.\quad+A_{1} \cos \theta_{1}+A_{2} \cos \theta_{2}\right] e^{-1 \theta_{1}} d \theta_{1} d \theta_{2}
\end{aligned}
$$

where $\bar{W}$ is complex conjugate of $W$.

In this case the existence of the continuous in $t$ and quasiperiodic solution of the equation (3.1) is determined. We can find therefore the solution of (3.3) in the form

$$
\begin{gathered}
\nu=\nu_{1} \varepsilon^{1 / 3}+\nu_{2} \varepsilon^{2 / 3}+\nu_{3} \varepsilon+\ldots \\
W=W_{1} \varepsilon^{1 / 3}+W_{2} \varepsilon^{2 / 3}+W_{3} \varepsilon+\ldots
\end{gathered}
$$

Substituting (3.4) into (3.3) we can find the system of equations determining the functions $\nu_{1}, \nu_{2}, \nu_{3}, \ldots$ and the quantities $W_{1}, W_{2}, W_{3}, \ldots$

$$
\begin{aligned}
\nu_{1} & =a_{1} e^{i \theta_{1}}+\bar{a}_{1} e^{-i \theta_{1}}, \quad W_{1}=0 \\
\nu_{2} & =a_{2} e^{i \theta_{1}}+\bar{a}_{21} e^{-i \theta_{1}}, \quad W_{2}=0 \\
\nu_{3} & =a_{3} e^{i \theta_{1}}+\bar{a}_{3} e^{-i \theta_{1}}+\frac{\gamma}{8 \nu_{1}^{2}}\left(a_{1}^{3} e^{3 i \theta_{1}}+\bar{a}_{1}^{3} e^{-3 i \theta_{1}}\right) \frac{A_{2}}{2\left(\nu_{1}^{2}-\nu_{2}^{2}\right)}\left(e^{i \theta_{2}}+e^{-i \theta_{2}}\right) \\
W_{3} & =3 \gamma a_{1}^{2} \bar{a}_{1}-\frac{A_{1}}{2}
\end{aligned}
$$

where $a_{i}(i=1,2,3, \ldots)$ are arbitrary constants, which are the terms of the power series

$$
a=a_{1} \varepsilon^{1 / 3}+a_{2} \varepsilon^{2 / 3}+a_{3} \varepsilon+\ldots
$$

$\bar{a}_{i}(i=1,2,3, \ldots)$ are complex conjugate of $a_{i}$. Giving $W=0$, i.e. $W_{3}=0$, $W_{4}=0, \ldots$ we can find the solution of equation (3.2). From this result we find 
the continuous in $t$ and quasiperiodic solution with frequency basis $\nu_{1}, \nu_{2}$ of the equation (3.1):

$$
\begin{aligned}
x(t, \varepsilon)= & \sqrt[3]{\frac{2 A_{1}}{3 \gamma}}\left(\cos \nu_{1} t-\sin \nu_{1} t\right) \varepsilon^{1 / 3}-\frac{2 \nu_{1}}{3 \sqrt[3]{18 A_{1} \gamma^{2}}}\left[\sin \nu_{1}(t+\Delta)\right. \\
& \left.+\cos \nu_{1}(t+\Delta)+2 \sin \nu_{1}(t-\Delta)+2 \cos \nu_{1}(t-\Delta)\right] \varepsilon^{2 / 3} \\
- & {\left[\frac{2}{9}\left(\frac{\nu^{2}}{\gamma A_{1}}+\frac{A_{1}}{16 \nu_{1}^{2}}\right)\left(\cos \nu_{1} t-\sin \nu_{1} t\right)\right.} \\
& +\frac{2 \nu_{1}^{2}}{9 \gamma A_{1}}\left(\cos \nu_{1}(t-2 \Delta)-\sin \nu_{1}(t-2 \Delta)\right) \\
& \left.+\frac{A_{1}}{24 \nu_{1}^{2}}\left(\cos 3 \nu_{1} t+\sin 3 \nu_{1} t\right)-\frac{A_{2}}{\nu_{1}^{2}-\nu_{2}^{2}} \cos \nu_{2} t\right] \varepsilon+\ldots
\end{aligned}
$$

This paper is completed with financial support of the Council for Natural Sciences of Vietnam.

\section{References}

1. Malkin I. G. Some problems of theory of nonlinear oscillations, Gostekhizdat 1956.

2. Le Xuan Can. On quasiperiodic solution of the nonlinear nonautonomous system with delay in the resonance case. Ukrain Journal of Maths, T.33, No $3,1981$.

Received November 2, 1997

\section{VỀ DAO ĐộNG TỰA TUẦN HOÀN CỦA HỆ ĐộNG LỤC PHI TUYẾN LIAPUNOV CÓ CHẬM}

Công trình đành cho việc nghiên cứu dao động tựa tuần hoàn của hệ động lực phi tuyến Liapunov có chậm. Đã nhận được các kết quả sau đây:

- Xác định điều kiện cần và đủ cho sự tồn tại nghiệm tựa tuần hoàn mô tả các quá trình dao động của hệ.

- Xây dựng nghiệm tựa tuần hoàn gần đúng dưới dạng chuối lũy thừa.

- Khảo sát dao động tựa tuần hoàn của hệ động lực phi tuyến có chậm loại Duffing với nhiễu loạn tuần hoàn. 